\title{
CHALLENGE IN THE TREATMENT OF TAKAYASU ARTERITIS MANIFESTATIONS WITH A HIGH BURDEN OF ATHEROSCLEROSIS
}

\author{
Jean Michell Correia Monteiro ${ }^{1, \star}$, Carlo Scognamiglio Renner Araújo ${ }^{1}$, Pablo Vinicius da Fonseca ${ }^{1}$, Marina Barguil Macedo \\ Diogo Souza Domiciano ${ }^{1}$, Samuel Katsuyuki Shinjo ${ }^{1}$
}

1.Universidade de São Paulo, São Paulo (SP), Brazil.

*Corresponding author:jk_michell@hotmail.com

\section{BACKGROUND}

The differentiation of disease activity and damage burden in Takayasu arteritis (TAK) is challenging. Symptoms are usually nonspecific and misleading, and differentiating progression of atherosclerotic lesions from active lesions can be difficult.

\section{CASE REPORT}

A 39-year-old woman was admitted to the emergency department with mesenteric angina. The patient was asymptomatic until intermittent abdominal pain began one month prior to admission. Abdomen computed tomography (CT) showed a fusiform aneurysm of the infrarenal aorta with multiple areas of diffuse vascular calcification and various strictures in the intestinal vasculature. She was referred to vascular evaluation. On examination there was absence of radial pulse, and a bilateral infraclavicular bruit were observed. Blood pressure could only be obtained in the lower limbs. Laboratory analysis showed acute renal injury and elevated acute phase reagents with negative blood cultures. Computed tomography angiography demonstrated stenosis of both carotids, the brachiocephalic trunk and the left subclavian arteries, as well as stenosis and thickening in superior mesenteric, celiac trunk and both renal arteries. She received methylprednisolone $1 \mathrm{~g} /$ day for 3 days and maintenance with prednisone $1 \mathrm{mg} / \mathrm{kg} /$ day plus methotrexate, with important clinical and laboratory improvement. One month later, she was hospitalized again for recurrence of abdominal pain, now associated with vertigo and intermittent claudication in both legs. New CT angiography suggested a new aneurism of the celiac trunk, and with elevated acute phase reagents. She was instructed to properly adhere to medication. Methylprednisolone $1 \mathrm{mg} / \mathrm{kg} /$ day was initiated, blood cultures were performed and a PET-CT was provided. Streptococcus viridans was isolated in two pairs of blood cultures, and ceftriaxone was started. Transesophageal echocardiography and dental evaluation were unremarkable, and intestinal translocation was suspected. The PET-CT results showed a localized uptake in the region suggested of the new aneurysm. A multidisciplinary meeting with vascular surgery, rheumatology, infectiology and radiology was conducted. According to discussion, there was no new aneurysm but an area of amorphous tissue around the celiac trunk with several areas of dilation and strictures that were reduced compared to previous CT angiography. Disease activity in the context of poor adherence was suspected, but without new severity criteria for new methylprednisolone pulse therapy. Treatment was maintained with clinical improvement, and she was discharged for outpatient follow-up. Three months later, the patient had maintained well with no complaints regarding glucocorticoid reduction.

\section{CONCLUSION}

Takayasu arteritis can represent a very difficult challenge to treat and adjust immunosuppression. Care regarding adherence and differential diagnosis are essential to avoid possible interventions with potential for iatrogenicity.

\section{KEYWORDS}

Takayasu arteritis, Atherosclerosis, Vasculitis. 